\title{
ARIMA vS. MACHINE LEARNING IN TERMS OF EQUITY MARKET FORECASTING
}

\author{
Iulian-Cornel LOLEA ${ }^{1}$, Ioan-Radu PETRARIU², Adriana GIURGIU³
}

\author{
${ }^{1}$ Bucharest University of Economic Studies, Bucharest, Romania \\ ${ }^{2}$ Bucharest University of Economic Studies, Bucharest, Romania \\ ${ }^{2}$ Bucharest University of Economic Studies, Bucharest, Romania; Department of \\ International Business, Faculty of Economic Studies, University of Oradea, \\ Oradea, Romania \\ loleaiulian@gmail.com \\ radu.petrariu@rei.ase.ro \\ adrianagiurgiu@gmail.com
}

\begin{abstract}
Through this paper we aimed to develop a comparison between ARIMA, Prophet, KNN and Neural Networks in terms of stock prices forecasting. After reviewing the literature, we noticed that there is a plethora of studies that address this problem of forecasting, but very few have made comparisons that include ARIMA, machine learning, but also the Prophet forecasting model developed by Facebook, which brought interesting results for certain data series. Based on methodologies validated by other authors, we compared these models in our paper and we sought to obtain promising results regarding performance evaluation. The comparison was made in-sample, the training period being 01/01/2010 $31 / 07 / 2021$, but also out-of-sample (01/08/2021 - 31/10/2021). The study was performed for Societe Generale's stock, using daily observations. Statistical loss functions such as RMSE, MPE, MAPE, MAE, and ME were used for comparison. The results indicated an outperformance of Neural Networks, both in-sample and out-of-sample, this model being on the 1st place according to the aggregated score. It is also noteworthy that the ARIMA model was in second place in-sample, ahead of KNN, but for out of sample these two algorithms changed their positions. On the other hand, the Prophet algorithm performed the weakest, both in-sample and out of sample. Also, we must underlie that all four algorithms had a clear tendency to overestimate the price of Societe Generale, according to the results of the statistical loss functions ME and MPE. Finally, it should be noted that the results were consistent with what other authors found out, especially for the out-ofsample period, where the machine learning models performed best.
\end{abstract}

Keywords: loss functions; machine learning; autoregressive; equity markets.

JEL Classification: G17; G11; C22; C38.

\section{Introduction}

Time series are difficult to model, especially due to time variability and seasonality. However, over time, plenty models and methodologies have been developed aiming to model time series, and here we can include forecasting stock prices. This objective is a very difficult one, but there are different papers that have set out to 
achieve this, obtaining gratifying results, especially when machine learning algorithms are used for the in-sample period.

In the literature there are a plethora of papers that have addressed this issue of stock price forecasting, and some of these articles have been used to substantiate this paper. There are articles that address classical statistical models, machine learning models, but also articles that make comparisons between the two types of approaches. In most cases, the results indicated an over-performance of machine learning models, especially when talking about in-sample evaluation.

In this article we set out to present a comparison on the performance of ARIMA, Prophet, KNN and Neural Networks for estimating the share price of Societe Generale. The comparison will be made based on the statistical loss functions, while the models will be ranked based on an aggregate score for all the criteria. Also, we will use indicators to show whether there is a bias for the models in terms of overestimating or underestimating the share price.

The methodology used in the study is like that used by other authors such as Chan (2020) or Madhuri et al. (2020) in terms of comparisons between models. This methodology has been extended to KNN and Neural Networks. The results obtained were in line with what was observed in the literature, indicating an overperformance of Neural Networks, both in-sample and out-of-sample. On the other hand, during the in-sample period, a good performance of the ARIMA model was also observed, this model ranked second among the four models considered in this paper.

The results of this research are important and can be used as part of a trading strategy, both by retail investors and by institutional investors. Also, there are possibilities to improve and refine the models such as considering an HMM methodology, in addition to machine learning algorithms.

\section{Literature review}

Forecasting the stock prices is a complex activity, which involves a thorough research of the literature. To substantiate this paper, we started from articles that address classical statistical methodologies and we continued with some papers that address various machine problems in terms of use for stock market forecasting. We also tried to identify articles in which a comparison was made between these two generations of models.

The problem of forecasting has been addressed in recent decades in various papers, but among the articles that have added to the area of classical prediction models I can mention the work of Gencay (1996) which addressed the problem of a prediction using different rules of moving averages. This type of analysis is an incipient one, and will then be developed by Gencay and Stengos (1998) who brought an additional analysis in this area of the use of moving averages for prediction. Also, the technique was later significantly improved by Wei et al. (2014), combining several methodologies to achieve significant results compared to a buy and hold strategy.

Classical statistical analysis was further improved by researches based on ARIMA models. Important for substantiating the methodology in this paper are the article published by Banerjee (2014), for the Indian stock market, and Mondal et al. (2014). The authors of the two papers managed to calibrate ARIMA models for the 
analysed data series, used the AIC and BIC informational criteria to choose the most appropriate model and obtained conclusions regarding the performance of these models according to different forecast horizons and for different time periods. At the same time, Mondal et al. (2014) highlighted the need to study ARIMA models for stocks within the same sector of activity, but also within different sectors of activity, being observed different performances depending on the type of company and sector. Other important papers that considered the prediction based on ARIMA are those of Ariyo et al. (2014) or Jarrett and Kyper (2011). The methodologies used in all these papers were the basis for the current study.

In this paper we aimed to make a performance comparison regarding stock prices forecasting between ARIMA, Prophet, KNN regression and Neural Networks. Thus, another important paper that was used to substantiate the methodology is that of Chan (2020) in which he made a comparison between the performance of ARIMA and Prophet for the equity market in Myanmar. Moreover, we should consider the research of Madhuri et al. (2020), which was made in the same sense, but also the article by Satrio et al. (2021) which compared the performance of the two methodologies during the crisis generated by the Covid-19 Pandemic. On the other hand, we considered important the paper of Fang et al. (2019) in which they compare the performance of the Prophet model with that of some machine learning algorithms.

Researches on the use of machine learning algorithms for stock price forecasting are not few, and among them we used as a research base Milosevic's (2016) article, which addresses a prediction problem from the perspective of a long-term investment. Close to our methodology is the research of Ban et al. (2013), but also that of Alkhatib et al. (2013), in which the KNN algorithm is applied in different forms for realizing a forecast regarding the stock market. Also, we considered the article of Vaisla and Bhatt (2010), but also that of Pang et al. (2020), in which the neural networks methodology is applied for forecasting.

\section{Methodology and data}

The methodology for this paper involves the implementation of four stock prices forecasting models: ARIMA, Prophet, KNN regression and Neural Network. These algorithms will be applied for the stock's price of Societe Generale.

For this stock prices we have obtained daily prices from public data sources for the period between: 01/01/2010 - 31/10/2021

This period was divided into two sub-periods:

In-sample (training period): 01/01/2010 - 31/07/2021

Out-of-sample period: - 01/08/2021 - 31/10/2021

For each of these periods, indicators such as RMSE, MAD, MSE or others will be. For each of these periods, indicators such as RMSE, MAD, MSE or others will be calculated for model errors and comparisons will be made. It is important to make a comparison for both the in-sample period and the out-of-sample period, in order to obtain the most relevant conclusions.

To estimate the ARIMA model, the Box-Jenkins methodology will be initially applied. In order to choose the most suitable model, the auto.arima function applied in RStudio will be used, this being based on an iterative selection process. 
The Prophet model is developed by Facebook to make predictions about data series. It will be used for forecasting, taking into consideration its three components: i) $g(t)$ that models the trend ii) $s(t)$ that models the seasonality using Fourier series and iii) $h(t)$ that takes into account the effects days off or extreme events. Thus, the shape of the model is as follows:

$\mathrm{y}(\mathrm{t})=\mathrm{g}(\mathrm{t})+\mathrm{s}(\mathrm{t})+\mathrm{h}(\mathrm{t})+\epsilon_{\mathrm{t}}$

The trend parameter $(\mathrm{g}(\mathrm{t}))$ is responsible for shouldering two other parameters: growth saturation and trend change points. The growth saturation takes into account how consistent the data are (ie if an element that can change their dynamics has appeared)

The third algorithm is KNN. This is an algorithm that can be used both for classification and to solve regression problems, but more often it is used for classification problems. However, I will apply it for regression issues in order to be able to make an estimate regarding the price of the Societe Generale share. For this we will use the tsfknn package from Rstudio. It should be noted that to predict the new values in the data series the algorithm uses the so-called similarity of characteristics.

Another way to predict data is to use a neural network. In this sense, we decided to use the RStudio package called nnetar, and for this study we will use a neural network with a single layer in order to keep things simple. Later, this type of the model can be developed and improvements can be made.

In this model we proposed there is a single layer of nodes with input values that send information to a single layer with docks that contain registration fields. This model will use time series lag values as input data, obtaining a nonlinear autoregressive model.

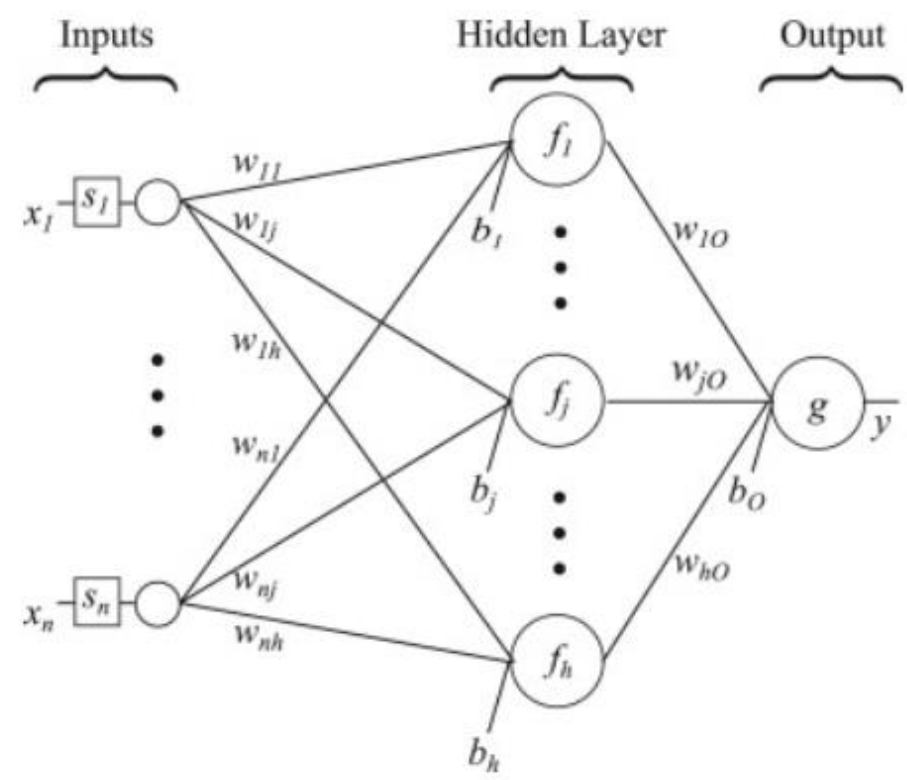

Figure 1: Neural Network with a single hidden layer

Source: Single hidden layer neural network - Nicola Manzini 
The number of hidden nodes was selected according to the following formula:

$$
N_{h}=\frac{N_{s}}{\left(\alpha *\left(N_{i}+N_{o}\right)\right)}
$$

Where,

$\mathrm{Ni}=$ the number of input neurons

$\mathrm{No}=$ the number of output neurons

$\mathrm{Ns}=$ the length of training sample

$\alpha=1.5^{-10}$

\section{Results}

The results of this research will be presented in two stages. In the first, the results that are obtained in terms of forecasting for ARIMA and neural networks, but also for the components of the Prophet model, will be presented in a graphical form. Second, the values obtained for the indicators by which the forecast errors are measured, both in-sample and out-of-sample, will be presented.

Following the estimation, we obtained results for each of the four methodologies. The ARIMA model was first estimated, and the iterative process in $\mathrm{R}$ indicated that an ARIMA model $(0,1,1)$ is the most suitable for the Societe Generale share data series.

The result of the forecast for Societe Generale shares using ARIMA $(0,1,1)$ is presented in graphical form below.

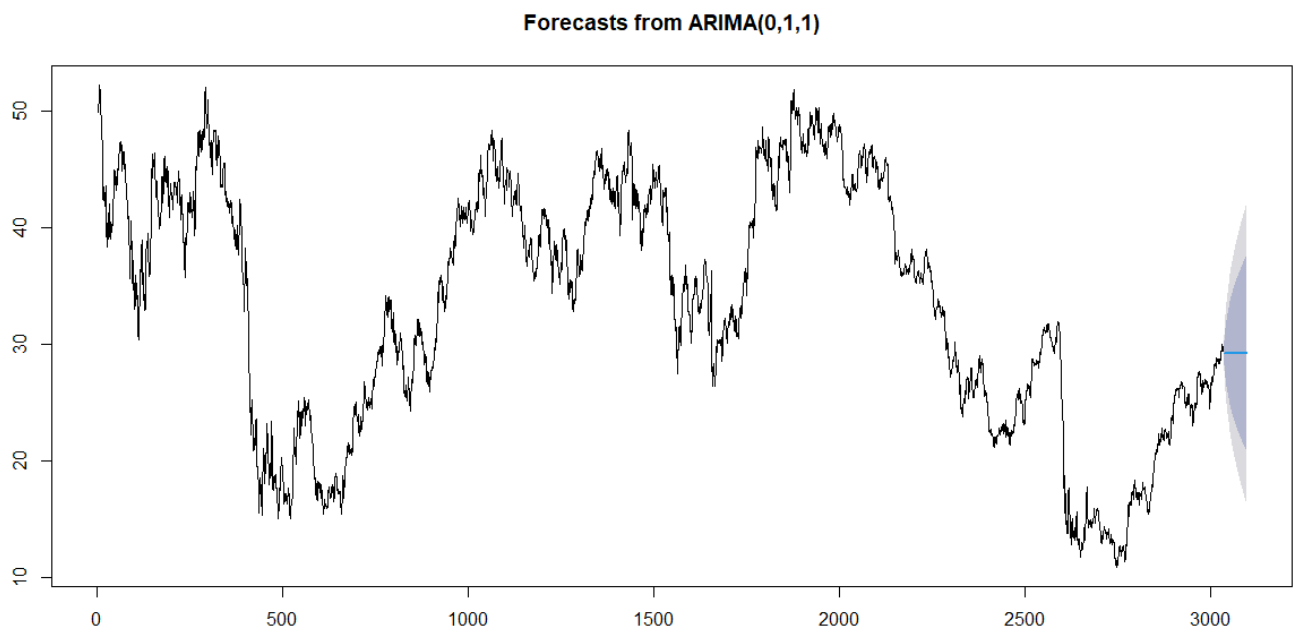

Figure 2: ARIMA forecast

Source: authors' own computation

Also, as we presented in the methodology part, for The Prophet, three components are estimated, and the results are presented below: 

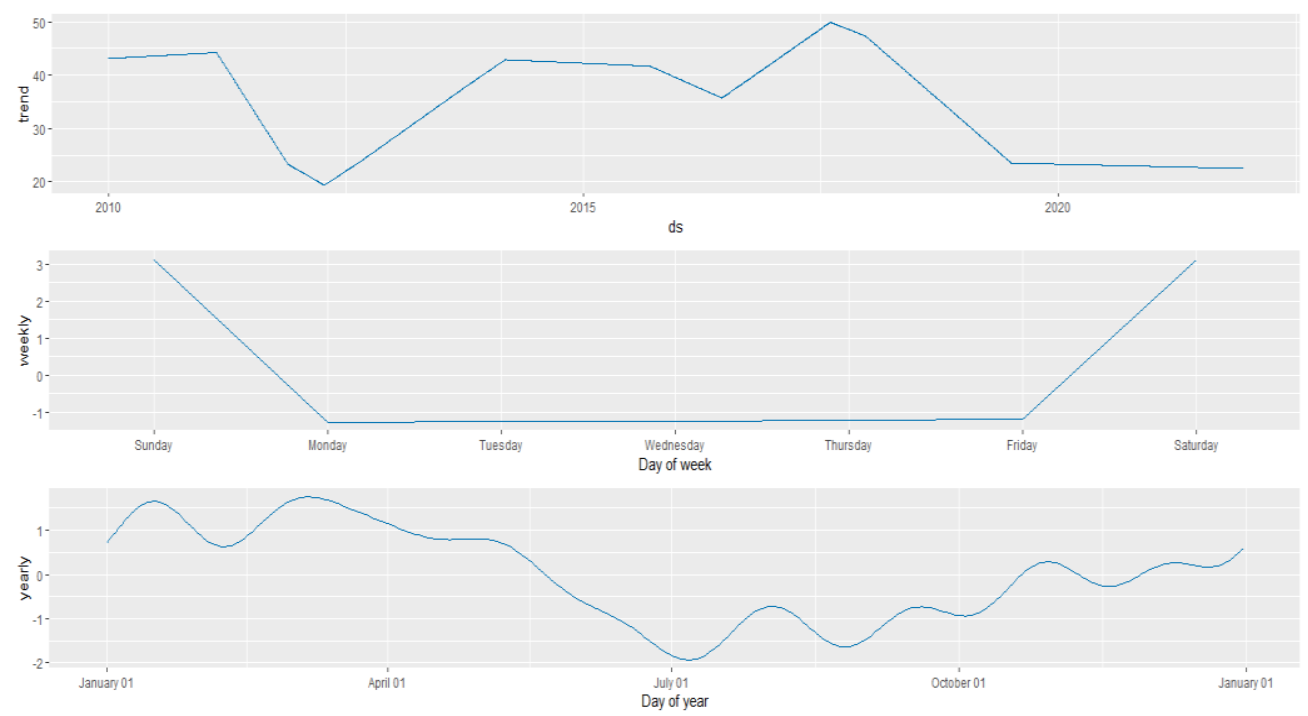

Figure 3. The three components of the Prophet Source: authors' own computations

In the same way as we did in the case of ARIMA, we will present the forecast obtained following the implementation of neural networks.

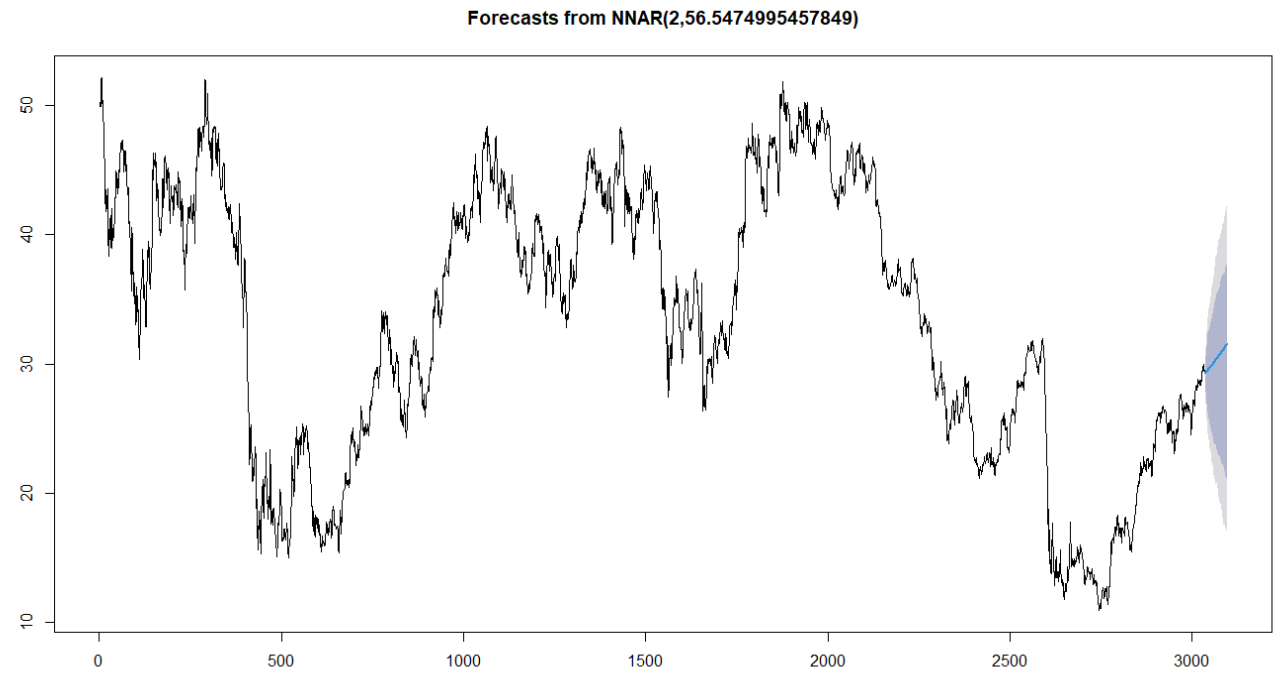

Figure 4: Neural Networks forecast

Source: authors' own computation 
Thus, it is possible to observe on the previous figure the estimated for the Societe Generale stock price, but also the confidence interval which is established with probabilities of $85 \%$ (dark blue) and $95 \%$ (light blue).

Further, we will present the results obtained for the indicators regarding the errors of the models for the training period. A rank was assigned to each indicator, so that an average rank could be calculated later

Table 1: Statistical loss functions - in sample

\begin{tabular}{|l|l|l|l|l|l|l|l|}
\hline \multicolumn{1}{|c|}{ Indicator } & & & & & & & \\
\hline Model & RMSE & RANK & MAE & RANK & MAPE & RANK & Average Rank \\
\hline ARIMA $(0,1,1)$ & 0.773 & 2 & 0.555 & 2 & 1.835 & 2 & 2.00 \\
\hline Prophet & 4.225 & 4 & 3.467 & 4 & 13.166 & 4 & 4.00 \\
\hline KNN regression & 2.751 & 3 & 2.158 & 3 & 7.511 & 3 & 3.00 \\
\hline Neural Network & 0.769 & 1 & 0.553 & 1 & 1.830 & 1 & 1.00 \\
\hline
\end{tabular}

Source: Authors' own computations

Neural network performed best for all indicators, and ARIMA was the second model, highlighting the fact that classical statistical models work well, at least for the in-sample period. On the other hand, the Prophet model performed the weakest, being on the 4th place for all three criteria.

Thus, the ARIMA model was very close to the in-sample performance of the machine learning models and it cannot be said that they strongly over-performed the classic statistical models, at least in-sample.

Below, we presented the values for the ME and PE indicators, which provide information regarding any potential estimation biases.

Table 2: ME si MPE for in-sample

\begin{tabular}{|c|c|c|}
\hline Model Indicator & $\mathrm{ME}$ & MPE \\
\hline ARIMA $(0,1,1)$ & -0.0064 & -0.050 \\
\hline Prophet & -0.0104 & -3.374 \\
\hline KNN regression & -0.0095 & -2.754 \\
\hline Neural Network & -0.009 & -0.075 \\
\hline
\end{tabular}

Source: authors' own computations

The values obtained for ME and MEP were negative for all cases, which shows that the models behave approximately the same in terms of estimation bias. Thus, it is about a systematic overestimation of the values of the Societe Generale stock's prices. The model error was calculated as the difference between the realized value and the estimated value.

After performing the in-sample analysis, we will also present the results obtained for the out-of-sample analysis. 
Table 3: Statistical loss functions - out-of-sample

\begin{tabular}{|l|l|l|l|l|l|l|l|}
\hline \multicolumn{1}{|c|}{ Indicator } & & & & & & $\begin{array}{l}\text { Average } \\
\text { Rank }\end{array}$ \\
\hline & RMSE & RANK & MAE & RANK & MAPE & RANK \\
\hline ARIMA $(0,1,1)$ & 2.981 & 3 & 2.785 & 3 & 8.341 & 2 & 2.67 \\
\hline Prophet & 4.357 & 4 & 4.984 & 4 & 16.050 & 4 & 4.00 \\
\hline KNN regression & 2.743 & 2 & 2.457 & 2 & 8.649 & 3 & 2.33 \\
\hline Neural Network & 1.015 & 1 & 0.984 & 1 & 2.743 & 1 & 1.00 \\
\hline
\end{tabular}

Source: Authors' own computations

The out-of-sample evaluation showed that the models based on machine learning (Neural Network and KNN regression) outperformed the classic ARIMA model, but also the Prophet. It is observed that KNN regression outperformed ARIMA $(0,1,1)$ for RMSE and MAE, while ARIMA $(0,1,1)$ was better for MAPE, but the result was quite close to that of KNN regression. On the other hand, Neural Network continued to be the best model, clearly outperforming the other models.

As was the case with in-sample, The Prophet model performed the poorest in terms of estimating the share price of Societe Generale. Moreover, it should be noted that all models obtained higher errors in the out-of-sample period than in the in-sample case, this result being in line with expectations.

\section{Conclusions}

Through this paper we tried to perform a comparison in terms of performance of classic models that can be used in stock prices forecasting and models based on machine learning algorithms. Also, we noticed that there are comparisons in the literature with the Prophet algorithm developed by Facebook and we considered it interesting to introduce it in the comparison that was made.

An in-sample and out-of-sample estimation was performed for ARIMA, Prophet, KNN regression and Neural Network. The aim was to forecast the price of the Societe Generale stock for the training period January 2010 - July 2021, so that the out-of-sample forecast can be made on a horizon of 3 months. For this scope, indicators such as RMSE, MAE, MAPE, ME or MPE were used, calculated for the models' errors.

The results for the in-sample estimation indicated that Neural Network obtained the best performance, being followed by the ARIMA model $(0,1,1)$. On the other hand, the Prophet model obtained the poorest performance for all the indicators considered. Thus, all these results indicated that in-sample, classical statistical models perform well enough compared to machine learning models. It should also be noted that all four models led to a systematic overestimation of the share price of Societe Generale.

Out-of-sample testing revealed better results for models based on machine learning. Neural Network continued to be the best model, and the Prophet to be the weakest, obtaining the highest values for all calculated loss function functions.

The results obtained in this paper are gratifying and show that the machine learning models add value and lead to better results than the classic statistical models, both in-sample and out-of-sample. On the other hand, it cannot be said that they over-performed by far, with the possibility that if testing is applied on 
another asset class or on other series with different volatility, a different ranking will be obtained. This could also be a way in which the research could expanded, along with the application of deep-learning algorithms.

\section{References:}

1. Alkhatib, K., Najadat, H., Hmeidi, I., \& Shatnawi, M. K. A. (2013) Stock price prediction using k-nearest neighbor (kNN) algorithm, International Journal of Business, Humanities and Technology, 3(3), 32-44.

2. Ariyo, A. A., Adewumi, A. O., \& Ayo, C. K. (2014, March) Stock price prediction using the ARIMA model, In 2014 UKSim-AMSS 16th International Conference on Computer Modelling and Simulation (pp. 106-112), IEEE.

3. Ban, T., Zhang, R., Pang, S., Sarrafzadeh, A., \& Inoue, D. (2013, November) Referential knn regression for financial time series forecasting, In International Conference on Neural Information Processing (pp. 601-608), Springer, Berlin, Heidelberg.

4. Banerjee, D. (2014, January), Forecasting of Indian stock market using timeseries ARIMA model, In 2014 2nd International Conference on Business and Information Management (ICBIM) (pp. 131-135), IEEE.

5. Chan, W. N. (2020) Time Series Data Mining: Comparative Study of ARIMA and Prophet Methods for Forecasting Closing Prices of Myanmar Stock Exchange, J. Comput. Appl. Res., 1, 75-80.

6. Fang, W. X., Lan, P. C., Lin, W. R., Chang, H. C., Chang, H. Y., \& Wang, Y. H. (2019, December) Combine Facebook prophet and LSTM with BPNN forecasting financial markets: the Morgan Taiwan Index, In 2019 International Symposium on Intelligent Signal Processing and Communication Systems (ISPACS) (pp. 1-2). IEEE.

7. Gencay, R. (1998), Non-linear prediction of security returns with moving average rules, Journal of Forecasting 15.3, 165-174.

8. Gencay, R. and Stengos, T. (1998) Moving average rules, volume and the predictability of security returns with feedforward networks, Journal of Forecasting, 17(5-6), 401-414.

9. Jarrett, J. E. and Kyper, E. (2011) ARIMA modeling with intervention to forecast and analyze Chinese stock prices, International Journal of Engineering Business Management, 3(3), 53-58.

10. Madhuri, C. R., Chinta, M. and Kumar, V. P. (2020, July) Stock Market Prediction for Time-series Forecasting using Prophet upon ARIMA, In 2020 7th International Conference on Smart Structures and Systems (ICSSS) (pp. 1-5). IEEE.

11. Milosevic, N. (2016) Equity forecast: Predicting long term stock price movement using machine learning, arXiv preprint arXiv:1603.00751.

12. Mondal, P., Shit, L. and Goswami, S. (2014) Study of effectiveness of time series modelling (ARIMA) in forecasting stock prices, International Journal of Computer Science, Engineering and Applications, 4(2), 13.

13. Pang, X., Zhou, Y., Wang, P., Lin, W. and Chang, V. (2020), An innovative neural network approach for stock market prediction, The Journal of Supercomputing, 76(3), 2098-2118. 
14. Satrio, C. B. A., Darmawan, W., Nadia, B. U. and Hanafiah, N. (2021), Time series analysis and forecasting of coronavirus disease in Indonesia using ARIMA model and PROPHET, Procedia Computer Science, 179, 524-532.

15. Vaisla, K. S. and Bhatt, A. K. (2010), An analysis of the performance of artificial neural network technique for stock market forecasting, International Journal on Computer Science and Engineering, 2(6), 2104-2109.

16. Wei, L. Y., Cheng, C. H. and Wu, H. H. (2014) A hybrid ANFIS based on nperiod moving average model to forecast TAIEX stock, Applied Soft Computing, 19, 86-92. 